\title{
Divergence of sun-rays by atmospheric refraction at large solar zenith angles
}

\author{
R. Uhl and T. Reddmann \\ Institut für Meteorologie und Klimaforschung, Forschungszentrum Karlsruhe, Germany \\ Received: 1 March 2004 - Published in Atmos. Chem. Phys. Discuss.: 16 April 2004 \\ Revised: 17 August 2004 - Accepted: 18 August 2004 - Published: 25 August 2004
}

\begin{abstract}
For the determination of photolysis rates at large zenith angles it has been demonstrated that refraction by the earth's atmosphere must be taken into account. In fact, due to the modified optical path the optical transmittance is thereby increased in most instances. Here we show that in addition the divergence of sun-rays, which is also caused by refraction but which reduces the direct solar irradiance, should not be neglected. Our calculations are based on a spherically symmetric atmosphere and include extinction by Rayleigh scattering, ozone, and background aerosol. For rays with $10 \mathrm{~km}$ tangent altitude the divergence yields a reduction of about $10 \%$ to $40 \%$ at solar zenith angles of $91^{\circ}$ to $96^{\circ}$. Moreover, we find that the divergence effect can completely cancel the relative enhancement caused by the increase of transmittance.
\end{abstract}

\section{Introduction}

It is well known that refraction by the earth's atmosphere may be important for photochemical calculations near the terminator. For example, Anderson and Lloyd (1990) and DeMajistre et al. (1995) present detailed calculations to the effect of refraction on the optical path. Besides the lengthening of the sunlit day, at large solar zenith angles $\left(>90^{\circ}\right)$ the inclusion of refraction reduces the optical depth of the direct beam in most cases, and therefore the radiation is enhanced. DeMajistre et al. (1995) and Trentmann et al. (2003) show substantial enhancement of photodissociation frequencies at large solar zenith angles for substances absorbing in the visual part of the spectrum as $\mathrm{O}_{3}$ and $\mathrm{NO}_{3}$, for instance. Balluch and Lary (1997) quantify the effect of refraction on stratospheric chemistry for a seven day period and find

Correspondence to: T. Reddmann

(thomas.reddmann@imk.fzk.de) changes of concentration for many substances in the order of several percent.

As it is shown in Fig. 1, there is another effect of refraction on the solar irradiance: as sun-rays reaching deeper layers in the atmosphere are more refracted, refraction causes a divergence of the direct solar beam, which is related to the apparent flatness of the solar disc. This additional divergence effect reduces the direct irradiance in the atmosphere and therefore competes against the enhancement caused by the changed optical path. In order to quantify this effect, which is not included in the studies cited above, we have performed ray tracing calculations in the visual part of the spectrum for large solar zenith angles, which account for both the change of optical path and divergence effect.

\section{Ray tracing}

\subsection{Refracting atmosphere}

A spherically symmetric atmosphere is taken as a basis. The mass density of air, $\varrho$, depending on (geometric) altitude $z$ is taken from the MSIS model of Hedin (1991) (official release NRLMSISE-00) for four typical atmospheric conditions, in order to test the influence of the temperature profile. The corresponding input data (besides the $10.7 \mathrm{~cm}$ solar flux indices equal to 150 and the planetary magnetic Ap indices equal to 4 ) and temperature profiles are shown in Fig. 2.

The refractive index $n_{\mathrm{S}}$ for standard sea-level air with mass density $\varrho_{\mathrm{s}}=1.225 \mathrm{~kg} / \mathrm{m}^{3}$ is taken from Edlén (1966) (Eq. 1), whereas for fixed wavelength the refractivity $n-1$ is assumed to be just proportional to $\varrho$, i.e.

$n-1=\frac{\left(n_{\mathrm{s}}-1\right) \varrho}{\varrho_{\mathrm{s}}}$

cf. again Edlén (1966). Finally, we use earth's radius $r_{0}=6370 \mathrm{~km}$. 


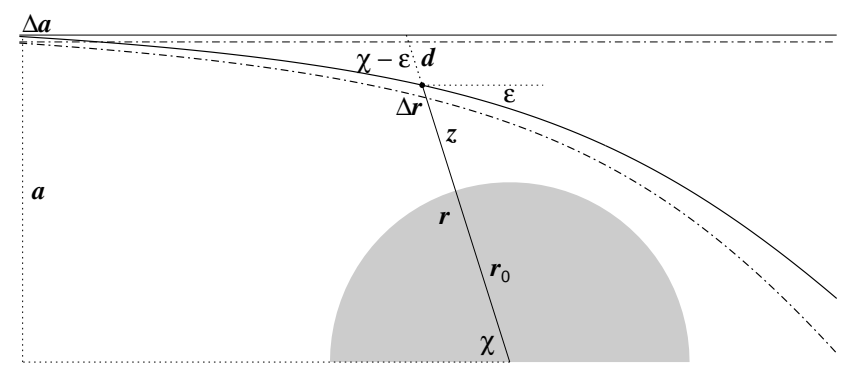

Fig. 1. Two neighbouring rays including/neglecting refraction.

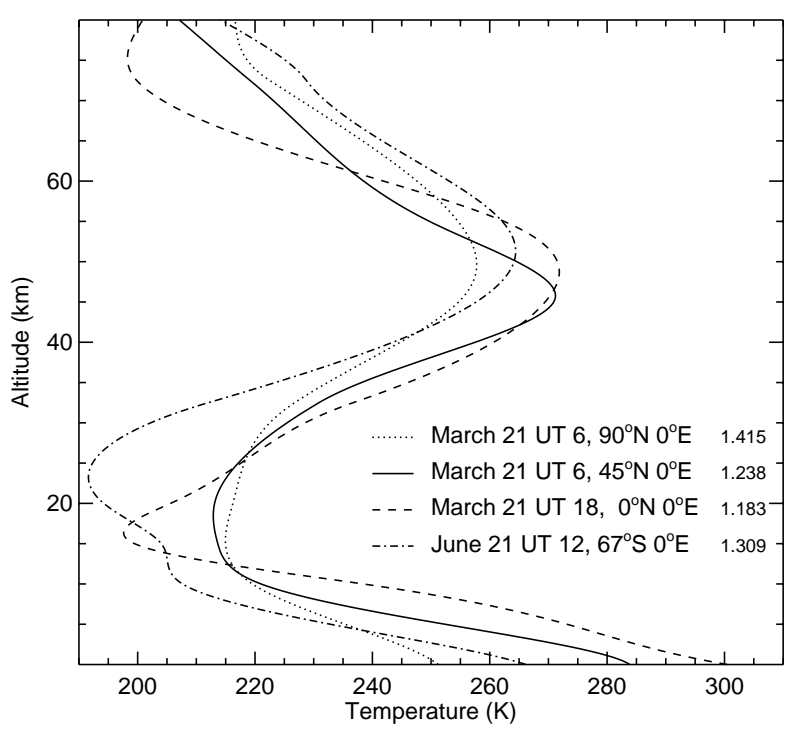

Fig. 2. The temperature profiles of the four used mass density profiles of air. At the right margin the mass density at altitude 0 is quoted in $\mathrm{kg} / \mathrm{m}^{3}$.

\subsection{Refraction equations}

We describe a single refracted sun-ray by the distance $r=r_{0}+z$ to the earth's centre and the deviation $\varepsilon$ from its original direction, both depending on the solar zenith angle $\chi$ as the independent variable; see Fig. 1.

To characterise the curvature of the ray at any point with $\chi=\chi_{0}$, we suppose temporarily that the atmosphere is horizontal plane parallel there. Logarithmic differentiation of Snell's law $n \sin \left(\chi_{0}-\varepsilon\right)=$ constant gives

$\frac{1}{n} \frac{d n}{d \chi}-\cot (\chi-\varepsilon) \frac{d \varepsilon}{d \chi}=0$

at $\chi=\chi_{0}$. This equation holds also for the spherically symmetric atmosphere (and hence along the whole ray) for the following reason: If the actual point of the ray and the actual ray's direction is fixed, then, in any case, the ray's curvature depends only on $n$ and $\operatorname{grad} n$, and therefore so does $d \varepsilon / d \chi$, whereas the other term $d n / d \chi$ in Eq. (1) depends on $\operatorname{grad} n$ alone. Thus, choosing the horizontal plane parallel atmo-

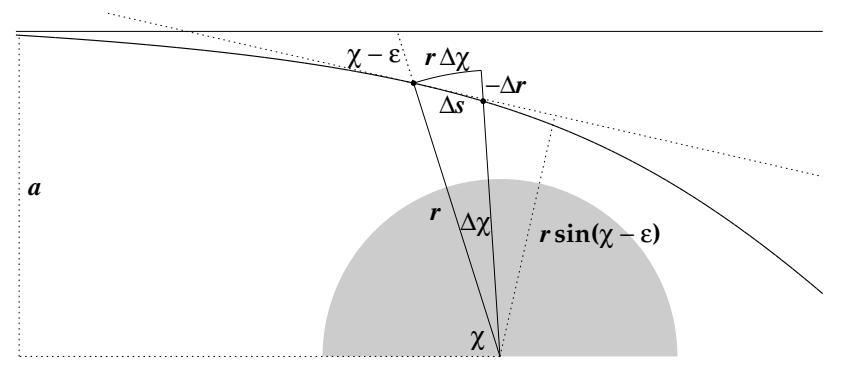

Fig. 3. Sketch to Eq. (2): $-\Delta r /(r \Delta \chi) \approx \cot (\chi-\varepsilon)$, where $\Delta r$ is induced by $\Delta \chi$ here, to Eq. (5): $r \Delta \chi / \Delta s \approx \sin (\chi-\varepsilon)$, and to the formula for the distance of earth's centre to the tangent.

sphere such that it has the same refractive index $n$ and the same $\operatorname{grad} n$ at the actual point as the spherically symmetric atmosphere, $d \varepsilon / d \chi$ and $d n / d \chi$ are the same in both cases. Dividing by the equation

$-\frac{1}{r} \frac{d r}{d \chi}=\cot (\chi-\varepsilon)$

(see Fig. 3), we obtain the basic equation

$\frac{d \varepsilon}{d \chi}=-\frac{r}{n} \frac{d n}{d r}$.

Moreover, we use the fact that the quantity

$a=n r \sin (\chi-\varepsilon)$

is constant on each refracted ray. In fact, logarithmic differentiation yields

$\frac{1}{a} \frac{d a}{d \chi}=\frac{1}{n} \frac{d n}{d \chi}+\frac{1}{r} \frac{d r}{d \chi}+\cot (\chi-\varepsilon)\left(1-\frac{d \varepsilon}{d \chi}\right)=0$

by Eqs. (1) and (2). Cf. also Born and Wolf (1980) (§3.2 Eq. 7), for instance. As is indicated in Figs. 1 and 3, $a$ is the distance of earth's centre to the corresponding unrefracted ray. For the distance of earth's centre to the tangent of the refracted ray (see Fig. 3) is equal to $r \sin (\chi-\varepsilon)=a / n \rightarrow a$ as $\chi \rightarrow 0$.

\subsection{Integration}

Note that if $a$ is specified, $r$ can be calculated to given $\chi$ and $\varepsilon$ via Eq. (4) by, for instance, iterating the fixed point equation

$r=\frac{a}{n(r) \sin (\chi-\varepsilon)}$,

starting with the approximation $r=a / \sin (\chi-\varepsilon)$. Thus we may consider Eq. (3), where the right hand side depends on $r$, as an ordinary differential equation for $\varepsilon(\chi)$. The approximation $\varepsilon=0$ at smaller $\chi$ (before the ray penetrates the deeper atmosphere) may be taken as initial value. 


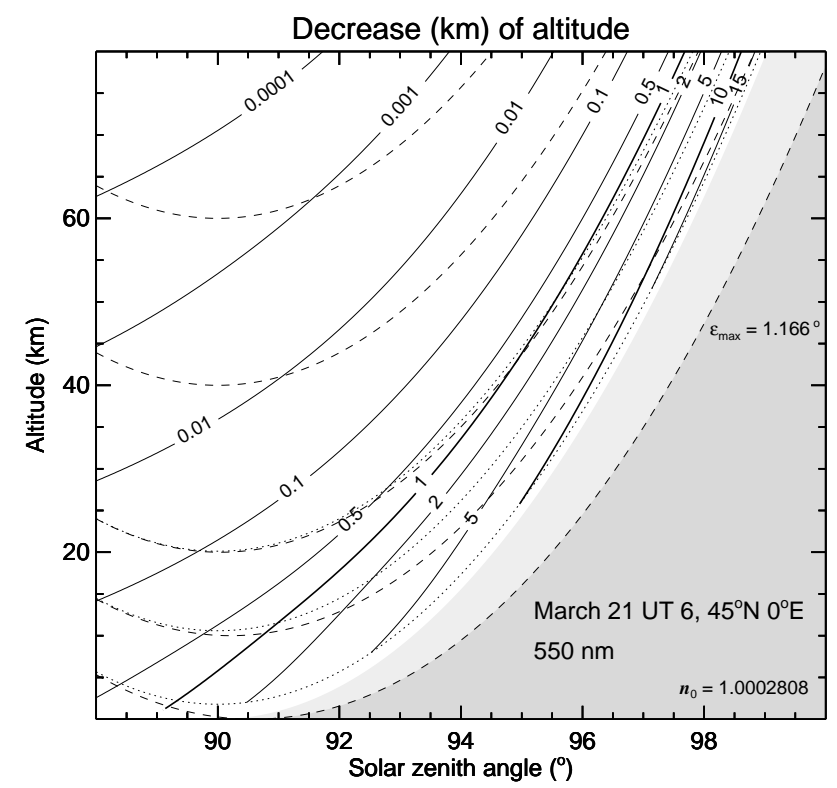

Fig. 4. The refraction induced decrease $d(\mathrm{~km})$ of altitude: each solid line consists of those points on unrefracted rays whose altitude is decreased by the indicated constant. The dashed lines are the refracted rays with tangent altitudes $0,10,20,40,60 \mathrm{~km}$. Three of the corresponding unrefracted rays are dotted. The dark/total shaded area shows the earth's shadow including/neglecting refraction. In addition, the refractive index $n_{0}$ at altitude 0 and the final direction deviation $\varepsilon_{\max }$ of the ray with tangent altitude 0 are quoted. Note that the refraction increases the solar zenith angle of the terminator by $\varepsilon=\varepsilon_{\max } / 2$.

A numerical integration of this initial value problem for many values of $a$ by an Adams-Bashforth method, for instance, yields the corresponding refracted rays, of which some are indicated in Fig. 4 for wavelength $550 \mathrm{~nm}$. This figure shows the refraction induced decrease $d$ of altitude, which is given by

$d=\frac{a}{\sin \chi}-r$

as in Fig. 1.

\section{Reduction of direct irradiance}

The direct beam is attenuated mainly by extinction in the atmosphere. However, the above mentioned divergence of rays reduces the direct (normal) irradiance, too. In the following we consider both attenuations, first separately, then the total reduction, and the impact of atmospheric refraction. Neglecting the angular extension of the solar disc, we assume that all sun-rays have the same original direction.

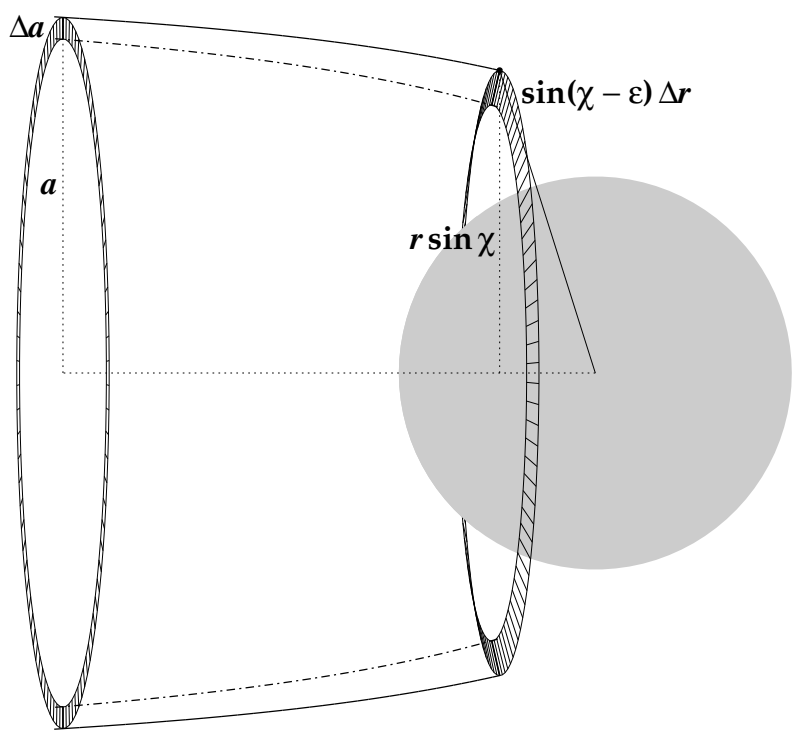

Fig. 5. The area ratio of the two hatched surfaces is nearly equal to the reduction factor $D$ of direct (normal) irradiance due to divergence.

\subsection{Divergence}

The divergence of neighbouring refracted rays as in Fig. 1 causes an attenuation of the direct (normal) irradiance. To quantify it, we consider two surfaces of revolution which are orthogonal to these rays (see Fig. 5): an annulus with radius $a$ and small width $\Delta a$, and the corresponding cone-like surface with radius $r \sin \chi$ and width $\sin (\chi-\varepsilon) \Delta r$, where $\Delta r$ is induced by $\Delta a$ now. According to Born and Wolf (1980) (§3.1 Eq. 31), for instance, the area ratio of both surfaces approximates the reduction factor $D<1$ of direct irradiance due to divergence,

$D \approx \frac{a \Delta a}{r \sin \chi \sin (\chi-\varepsilon) \Delta r}$,

or precisely,

$D=\frac{a}{r \sin \chi \sin (\chi-\varepsilon) \frac{\partial r}{\partial a}}=\frac{n}{\frac{\partial r}{\partial a} \sin \chi}$

(by Eq. 4), where $r$ is now regarded as a function of $\chi$ and $a$.

This reduction factor is calculated approximately with $\partial r / \partial a$ replaced by $\Delta r / \Delta a$ (with two neighbouring refracted rays as in Fig. 1) and plotted in Fig. 6 for wavelength $550 \mathrm{~nm}$, which is representative for the visual part of the spectrum in this study. As the refractivity $n-1$ for other wavelengths between $400 \mathrm{~nm}$ and $1000 \mathrm{~nm}$ differs by less than $2 \%$, this and the preceding figure would be nearly the same for every other wavelength in this range. 

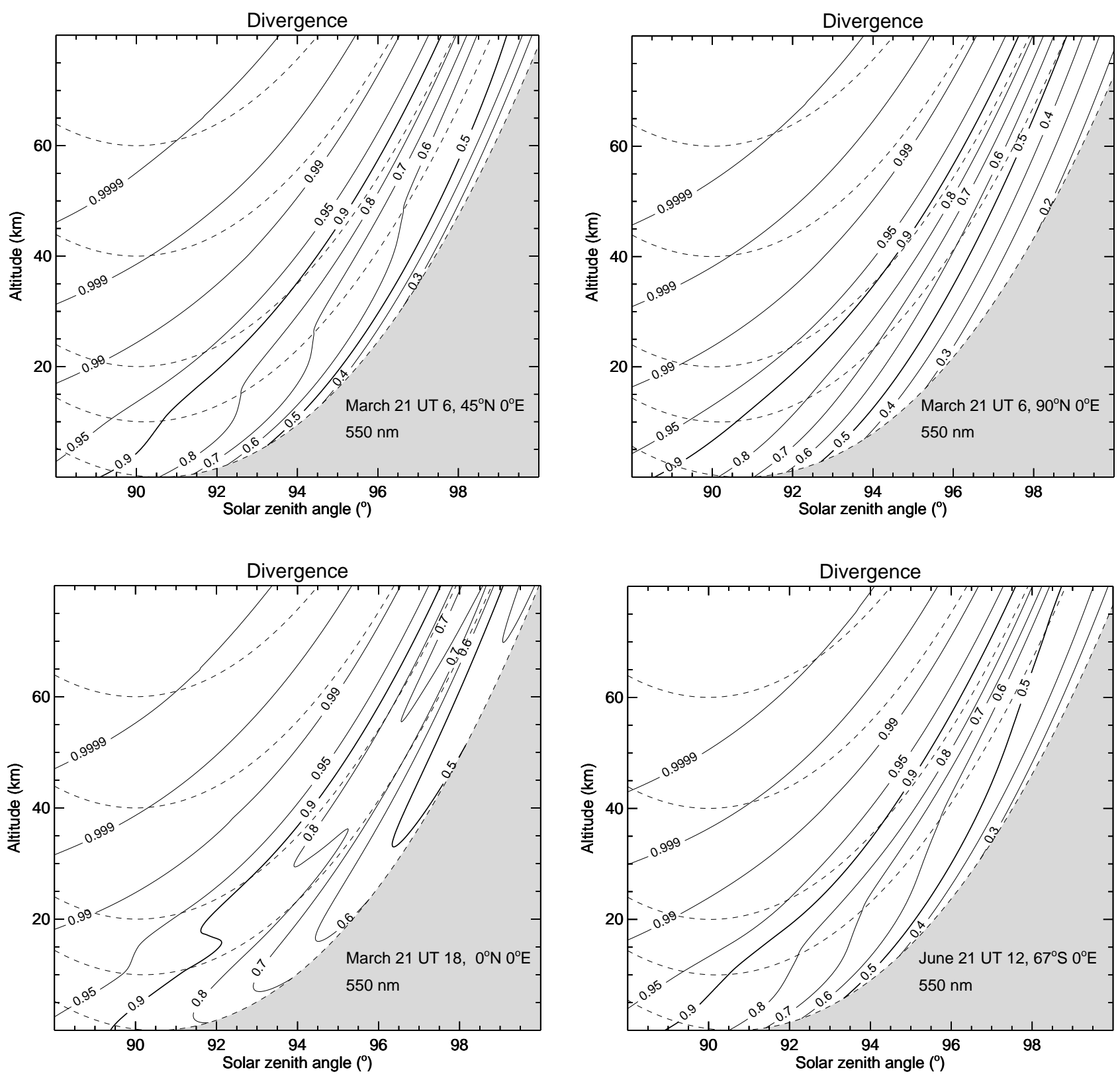

Fig. 6. The reduction factor $D$ of direct (normal) irradiance due to divergence for four atmospheric conditions: each solid line consists of the points where $D$ is equal to the indicated constant. The dashed lines are refracted rays as in Fig. 4. 


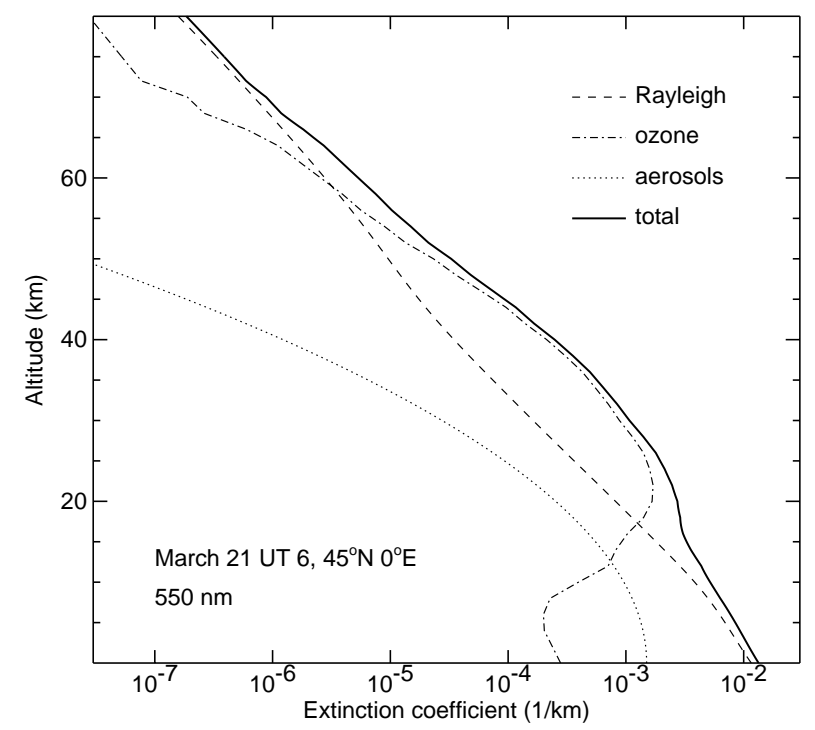

Fig. 7. The extinction coefficients $\alpha$ (Ray), $\alpha\left(\mathrm{O}_{3}\right), \alpha$ (aer), $\alpha$.

\subsection{Extinction}

We use the extinction coefficient $\alpha=\alpha$ (Ray) $+\alpha\left(\mathrm{O}_{3}\right)+$ $\alpha$ (aer) which is composed as follows; see also Fig. 7. The Rayleigh scattering coefficient $\alpha_{\mathrm{s}}$ (Ray) $=0.01149 / \mathrm{km}$ for wavelength $550 \mathrm{~nm}$ and standard sea-level air is taken from Bucholtz (1995) (Table 2), whereas for fixed wavelength the coefficient $\alpha$ (Ray) is assumed to be just proportional to mass density $\varrho$ of air, i.e. $\alpha($ Ray $)=\alpha_{\mathrm{s}}($ Ray $) \varrho / \varrho_{\mathrm{s}}$. Absorption by ozone is taken into account as $\alpha\left(\mathrm{O}_{3}\right)=\sigma\left(\mathrm{O}_{3}\right) N\left(\mathrm{O}_{3}\right)$ with the particle density profile $N\left(\mathrm{O}_{3}\right)$ of the U.S. Standard Atmosphere (1976) (Table 18, exponentially interand extrapolated) and with the constant cross section $\sigma\left(\mathrm{O}_{3}\right)=3.5 \cdot 10^{-25} \mathrm{~m}^{2}$ for wavelength $550 \mathrm{~nm}$; cf. Burrows et al. (1999). Finally, we use the simple aerosol extinction profile

$\alpha($ aer $)=0.0015 \mathrm{~km}^{-1} \exp \left(-\left(\frac{z}{15 \mathrm{~km}}\right)^{2}\right)$,

which corresponds approximately to background conditions in the lower stratosphere. Clouds are not taken into account.

The extinction reduces the direct radiation at any point by the factor

$T=\exp \left(-\int \alpha d s\right)$,

the transmittance, with a line integral along a refracted ray where

$\frac{d s}{d \chi}=\frac{r}{\sin (\chi-\varepsilon)}$

see Fig. 3. To evaluate the impact of refraction on the direct irradiance, also the transmittance in case of no refraction, $T_{\text {straight }}$, is calculated with a similar line integral to the same point but along an unrefracted ray.

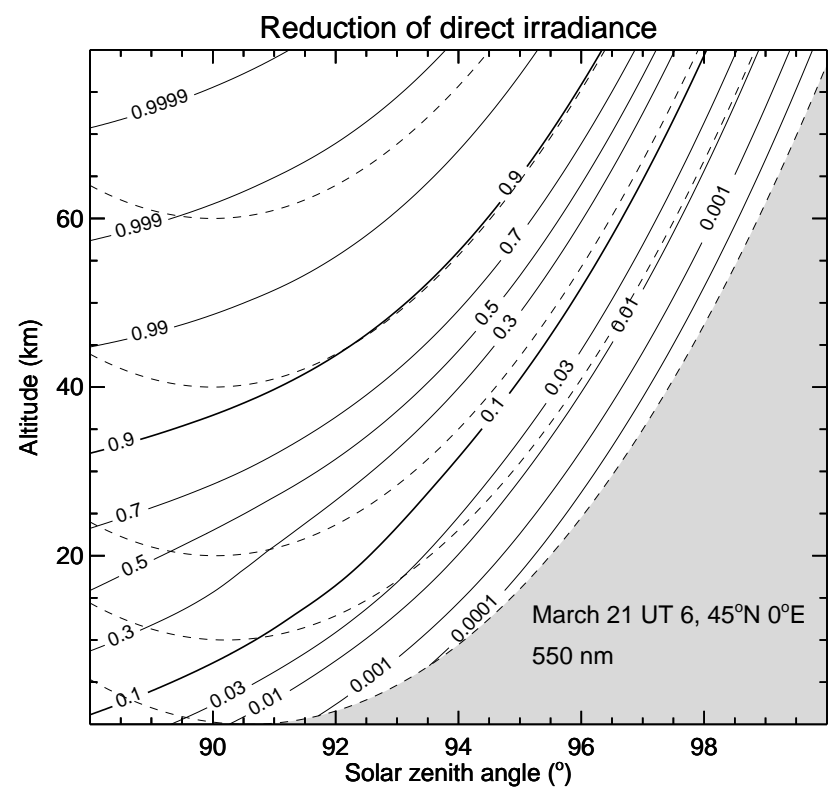

Fig. 8. The total reduction factor $D T$ of direct irradiance due to divergence and extinction (solid lines). The dashed lines are refracted rays.

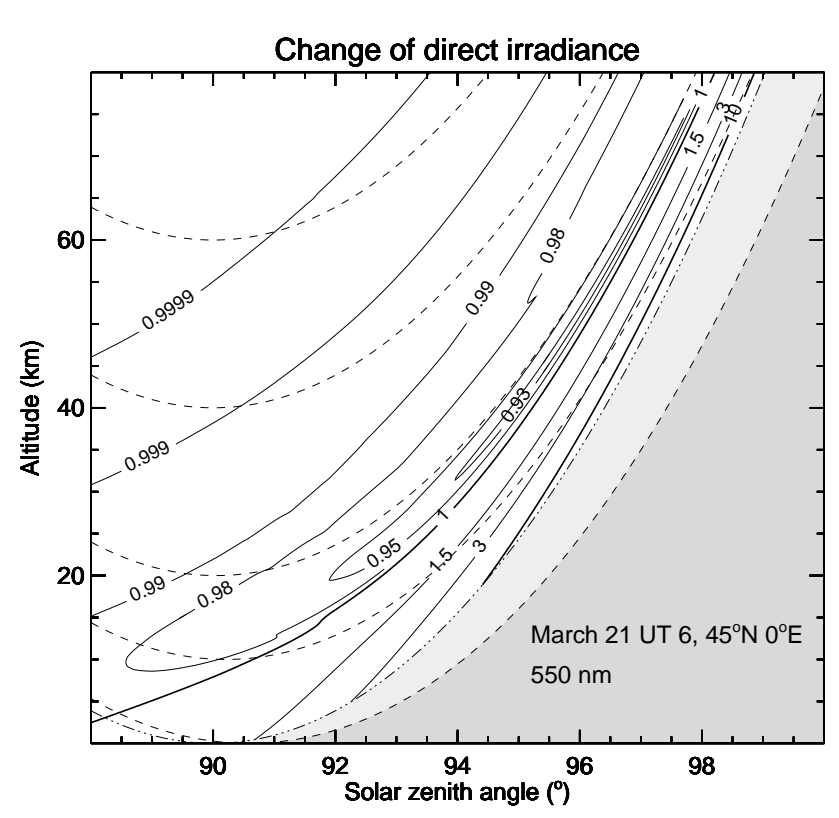

Fig. 9. The refraction induced changing factor $D T / T_{\text {straight }}$ of direct irradiance.

\subsection{Total reduction}

The irradiance of a direct beam is reduced simultaneously by divergence and extinction. Its total reduction factor $D T$ is plotted in Fig. 8. The influence of refraction on the direct irradiance is specified by the corresponding changing factor $D T / T_{\text {straight }}$ as shown in Fig. 9 . 


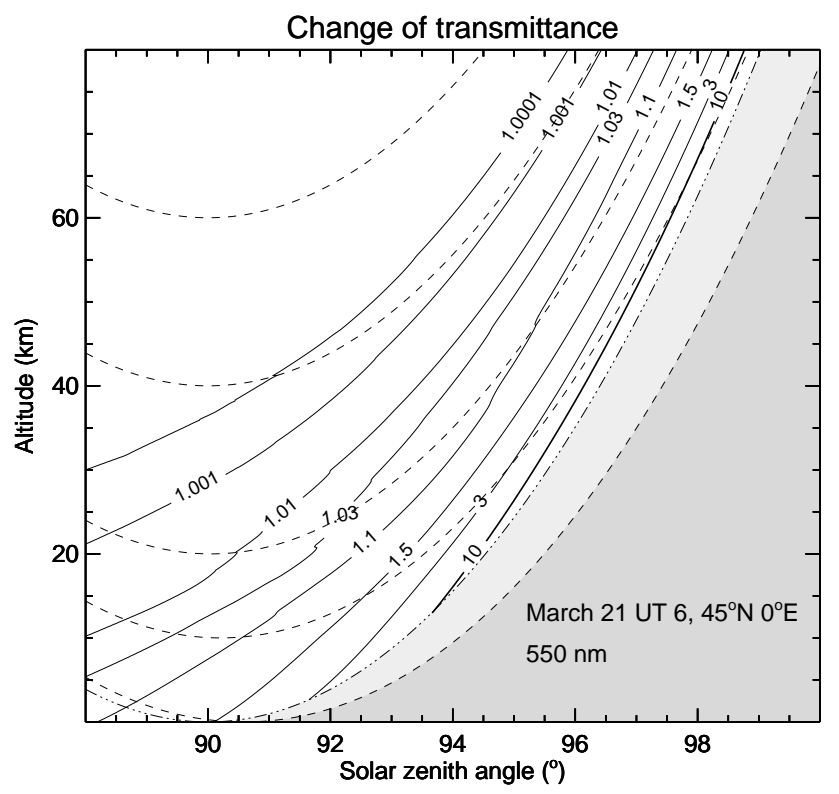

Fig. 10. The refraction induced changing factor $T / T_{\text {straight }}$ of transmittance.

For comparison also the refraction induced changing factor $T / T_{\text {straight }}$ of transmittance is plotted in Fig. 10. It describes the impact of refraction if the divergence effect is neglected. Note that at those points where $D T / T_{\text {straight }}<1$, the direct irradiance is more reduced by divergence than increased by change of transmittance.

\section{Results and discussion}

As shown in Fig. 6, the solar irradiance of the direct beam is substantially reduced by the divergence due to the atmosphere. Along rays with a tangent altitude of about $10 \mathrm{~km}$ and which are not affected by clouds or aerosol of tropospheric origin, the reduction exceeds $5 \%$ at about $90^{\circ}$ solar zenith angle and can reach about $40 \%$ at $96^{\circ}$ solar zenith angle. Comparing the results for the different meteorological conditions, the polar and equatorial temperature profiles for 21 March differ substantially. Obviously, the steep and highly variable temperature profile in the tropics disturbs the reduction field compared with the uniform decreasing irradiation in the case of polar spring. In general, along rays with $10 \mathrm{~km}$ tangent altitude the reduction exceeds $10 \%$ within a band of about $2^{\circ}$ from the terminator.

The total reduction caused by divergence and extinction, as shown in Fig. 8 for $550 \mathrm{~nm}$, is dominated by the extinction, as expected. For this wavelength Rayleigh scattering and ozone absorption essentially contribute to the extinction as follows from Fig. 7. For large solar zenith angles and deep rays the reduction of the direct beam is so high that scattered radiation may dominate the radiation field. Inspection of Fig. 8 of Dahlback and Stamnes (1991) shows that

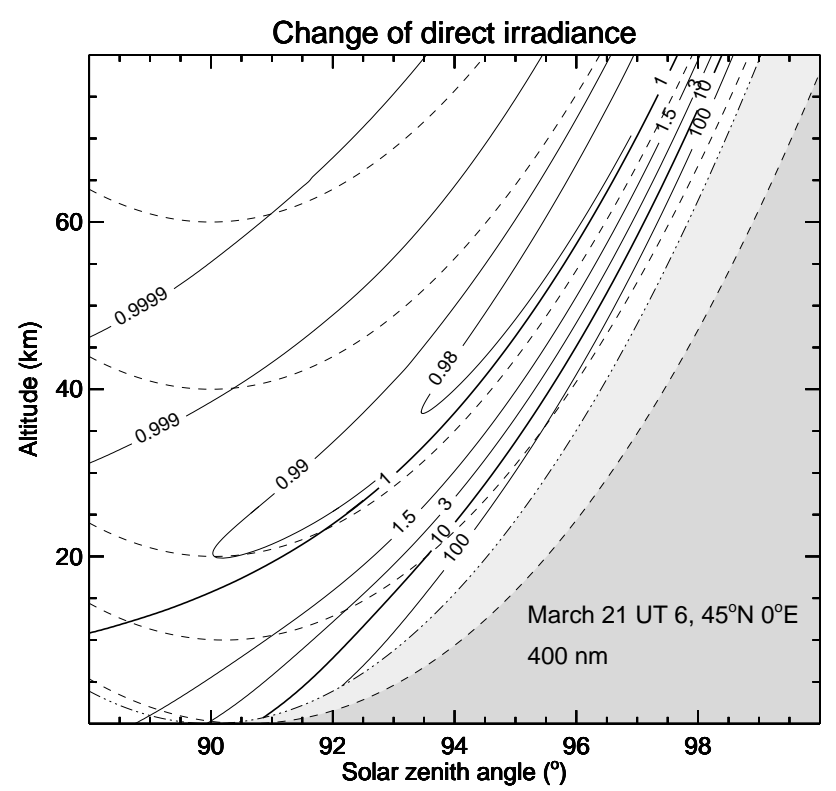

Fig. 11. Same as Fig. 9 but for wavelength $400 \mathrm{~nm}$.

about for a tangent altitude of $10 \mathrm{~km}$ the contribution of direct beam and scattered radiation are equal for wavelengths at $450 \mathrm{~nm}$ under clear sky conditions. As at $550 \mathrm{~nm}$ the extinction coefficient of Rayleigh scattering is only about half as large, we conclude that the direct beam dominates for rays with tangent altitude in the stratosphere. This is also in accordance with the photochemical calculations of DeMajistre et al. (1995).

We now turn to the question how the divergence effect compares with the change of transmittance caused by refraction. Again only rays with a tangent altitude higher than about $10 \mathrm{~km}$ need to be considered for the same reason as given above, so that Figs. 9 and 10 are sufficient for the discussion despite they do not show data at those points which are only met by refracted rays. In addition, these are rays which do not penetrate the troposphere and are not affected by clouds. Note that this is the part of the atmosphere where refraction causes a prolonged sunlit day. With that restriction, the band with an increase of the irradiance of more than $5 \%$ in Fig. 10 is more than halved by the divergence effect. In addition, radiation in the visual part of the solar spectrum is more important for photochemistry in the lower stratosphere where UV is mostly absorbed. Here the divergence effect narrows the band of enhancement even more. At about $20 \mathrm{~km}$ altitude and $93^{\circ}$ solar zenith angle, there is even a total reduction of more than $5 \%$.

Further calculations within the visual part of the spectrum show that at wavelengths greater than $550 \mathrm{~nm}$ the divergence effect counteracts the refraction induced enhancement of transmittance even more effective than just described. In particular, the region where divergence overcompensates the 
enhancement of transmittance (i.e. where $D T / T_{\text {straight }}<1$ ) sinks in comparison with that one in Fig. 9, e.g. by about 3 and $6 \mathrm{~km}$ for 600 and $750 \mathrm{~nm}$, respectively. Conversely, for 500,450 , and $400 \mathrm{~nm}$ this region is raised by about 3, 5, and $8 \mathrm{~km}$, respectively, and this holds approximately for the three surfaces $D T / T_{\text {straight }}=1.5,3,10$, too. Thus, at smaller wavelengths the divergence effect has a smaller impact.

For these five wavelengths we use the data $\alpha_{\mathrm{s}}$ (Ray) $\cdot \mathrm{km}$ $=0.008053,0.003261,0.01696,0.02616,0.04261$ and $\sigma\left(\mathrm{O}_{3}\right) \cdot 10^{25} \mathrm{~m}^{-2}=5,0.5,1,0.2,0.04$, respectively. Moreover, if altitude $z$ is fixed, $\alpha$ (aer) is assumed to be just proportional to $\exp (-\lambda / 400 \mathrm{~nm})$ for varying wavelength $\lambda$.

Fig. 11 shows the refraction induced changing factor $D T / T_{\text {straight }}$ of direct irradiance, just as Fig. 9 but for $400 \mathrm{~nm}$ especially. For even smaller wavelengths the portion of the diffusive part of the radiation field increases in the lower stratosphere, and the refractive effects become less important.

We point out that our previous calculations do not take into account the angular extension of the sun. This can be included by partition the solar disc into sufficiently small solid angles which can be considered as single sources of radiation. This results in some weighted averaging of any reduction factor $R(\chi, z)$ of direct irradiance as above with respect to a varying solar zenith angle $\chi$, according to the apparent solar diameter $2 \Delta \chi \approx 0.5^{\circ}$, but with fixed altitude $z$. More precisely, at any point with altitude $z$ and zenith angle of sun's centre, $\chi_{0}$, this averaging has the form

$$
\langle R\rangle\left(\chi_{0}, z\right)=\int_{\chi_{0}-\Delta \chi}^{\chi_{0}+\Delta \chi} R(\chi, z) W\left(\chi, \chi_{0}\right) d \chi .
$$

The nonnegative weighting function $W\left(\chi, \chi_{0}\right)$ describes the distribution of the solar radiation with respect to the "horizontal lines" of the solar disc. The most upper/lower part of the solar disc corresponds to the lower/upper limit of the integral, $\chi=\chi_{0} \mp \Delta \chi$, respectively.

In particular, the mean values $\langle D T\rangle$ and $\left\langle T_{\text {straight }}\right\rangle$, with $D$, $T, T_{\text {straight }}$ as introduced in Sec. 3, are the reduction factors of the direct irradiance (more precisely, of the direct actinic flux actually) accounting/neglecting refraction, both including the extension of the sun. The influence of refraction on the direct irradiance is now specified by the changing factor $\langle D T\rangle /\left\langle T_{\text {straight }}\right\rangle$.

Note that if the inequality $D T / T_{\text {straight }} \leq c$ holds with some constant $c$ in any interval of averaging, $\chi_{0}-\Delta \chi \leq \chi \leq \chi_{0}+\Delta \chi$, at a fixed altitude $z$, then also $\langle D T\rangle /\left\langle T_{\text {straight }}\right\rangle \leq c$ at the point $\left(\chi_{0}, z\right)$, as is seen by "averaging" the inequality $D T \leq c T_{\text {straight }}$. The similar implication with the inequalities $\geq c$ is also valid. Consequently, a plot of the ratio $\langle D T\rangle /\left\langle T_{\text {straight }}\right\rangle$ for $550 \mathrm{~nm}$, for instance, would be similar to Fig. 9: roughly speaken, the solid lines in this figure could not be displaced by more than $\Delta \chi \approx 0.25^{\circ}$. Likewise, a plot of the mean value $\langle D T\rangle$ would be similar to Fig. 8.

\section{Conclusions}

Our study of the refraction induced divergence effect of the direct solar irradiation in the visual part of the spectrum show that this effect should be included if refraction is considered at all in photochemical studies. For rays in the stratosphere and background aerosol conditions the divergence effect narrows the refraction induced enhancement to an inclined band, which shrinks as wavelength is increased from about 10 to $15 \mathrm{~km}$ thickness at $400 \mathrm{~nm}$ and which vanishs at about $800 \mathrm{~nm}$. Especially in the lower stratosphere, it may be justified to neglect refraction for photochemical calculation for larger wavelengths. Whereas high aerosol loading after volcanic eruptions would lead to a higher change of transmittance by refraction, the increase of scattering and the vertical filamentation probably makes special calculations necessary.

Acknowledgements. This work is a contribution to the project TEDDY (contract no. 6-056-0179) of the AFO 2000 framework and partly funded by the Bundesministerium für Bildung und Forschung.

Edited by: F.-J. Lübken

\section{References}

Anderson, D. E. and Lloyd, S. A.: Polar twilight UV-visible radiation field: perturbations due to multiple scattering, ozone depletion, stratospheric clouds, and surface albedo, J. Geophys. Res., 95, 7429-7434, 1990.

Balluch, M. and Lary, D. J.: Refraction and atmospheric photochemistry, J. Geophys. Res., 102, 8845-8854, 1997.

Born, M. and Wolf, E.: Principles of optics, Pergamon Press, 6th edn., 1980.

Bucholtz, A.: Rayleigh-scattering calculations for the terrestrial atmosphere, Appl. Opt., 34, 2765-2773, 1995.

Burrows, J. P., Richter, A., Dehn, A., Deters, B., Himmelmann, S., Voigt, S., and Orphal, J.: Atmospheric remote-sensing reference data from GOME - 2. Temperature-dependent absorption cross sections of $\mathrm{O}_{3}$ in the 231-794 nm range, J. Quant. Spectrosc. Radiat. Transfer, 61, 509-517, 1999.

Dahlback, A. and Stamnes, K.: A new spherical model for computing the radiation field available for photolysis and heating at twilight, Planet. Space Sci., 39, 671-683, 1991.

DeMajistre, R., Anderson, D. E., Lloyd, S., Swaminatham, P. K., and Zasadil, S.: Effects of refraction on photochemical calculations, J. Geophys. Res., 100, 18 817-18 822, 1995.

Edlén, B.: The refractive index of air, Metrologia, 2, 71-80, 1966.

Hedin, A. E.: Extension of the MSIS thermosphere model into the middle and lower atmosphere, J. Geophys. Res., 96, 1159-1172, 1991.

Trentmann, J., Bovensmann, H., Eyring, V., Müller, R. W., and Burrows, J. P.: Impact of accurate photolysis calculations on the simulations of stratospheric chemistry, J. Atmos. Chem., 44, 225240, 2003.

U.S. Standard Atmosphere, 1976: National Oceanic and Atmospheric Administration, National Aeronautics and Space Administration, United States Air Force, Washington, D.C., 1976. 